\section{Influência do consumo alimentar de ácidos graxos trans no perfil de lipídios séricos em nipo-brasileiros de Bauru, São Paulo, Brasil}

\author{
Dietary trans fatty acid intake and serum \\ lipid profile in Japanese-Brazilians in Bauru, \\ São Paulo, Brazil
}

\author{
Carla Novaes Bertolino 1 \\ Teresa Gontijo Castro ${ }^{1}$ \\ Daniela S. Sartorelli 1 \\ Sandra R.G. Ferreira ${ }^{2}$ \\ Marly Augusto Cardoso 1 \\ Grupo de Estudos de Diabetes \\ em Nipo-brasileiros 3
}

\author{
1 Faculdade de Saúde \\ Pública, Universidade de \\ São Paulo, São Paulo, Brasil. \\ 2 Escola Paulista de \\ Medicina, Universidade \\ Federal de São Paulo, \\ São Paulo, Brasil. \\ 3 Outros membros listados \\ no fim do artigo. \\ Correspondência \\ M. A. Cardoso \\ Departamento de Nutrição, \\ Faculdade de Saúde Pública. \\ Universidade de São Paulo. \\ Av. Dr. Arnaldo 715 , \\ São Paulo, SP \\ 01246-904, Brasil. \\ marlyac@usp.br
}

\begin{abstract}
This study describes the contribution of changes in trans fatty acid intake in relation to serum lipoproteins. A total of 328 Japanese-Brazilians of the first (Issei) and second (Nisei) generations, aged 40-79 years in 1993, were assessed in two cross-sectional surveys on health and nutritional status in 1993 and 2000. Multiple linear regression models were used with changes (2000/ 1993) in serum lipoproteins as the dependent variable and changes in dietary trans fatty acids (adjusted for total calories) as independent variable. In both genders a significant reduction was observed in total intake of trans fatty acids with lower LDL and increased HDL serum levels during 7-year follow-up. The mean intakes of trans fatty acids (\% of total energy) in 1993 and 2000 were: $5.1 \%$ and $3.4 \%$ for women and $4.7 \%$ and 3.3\% for men, respectively. Although the association between changes in dietary trans fatty acids and serum lipoprotein was not statistically significant, on average the intake of this trans fat was higher than the WHO recommendation (up to $1 \%$ of total energy).
\end{abstract}

Trans Fatty Acids; Lipids; Food Consumption

\section{Introdução}

A qualidade dos lipídios da dieta possui um papel importante no risco de desenvolvimento de diversas doenças crônicas. Estudos epidemiológicos prévios sugerem uma associação positiva entre consumo de ácidos graxos trans e ocorrência de doenças cardiovasculares em ambos os gêneros 1,2,3,4,5. O consumo deste tipo de gordura vem sendo apontado como um fator de risco importante tanto quanto o consumo de ácidos graxos saturados para o desenvolvimento de doenças cardiovasculares em ambos os gêneros 1,2,3,4,5,6.

Os ácidos graxos trans, isômeros geométricos e de posição dos ácidos graxos insaturados, podem ocorrer naturalmente em produtos derivados da carne e leite de animais ruminantes. Entretanto, as principais fontes de ácidos graxos trans na alimentação são os óleos vegetais parcialmente hidrogenados, contribuindo com cerca de 80 a $90 \%$ de todos os isômeros trans provenientes da dieta. Constituem fontes importantes de ácidos graxos trans na dieta: gorduras vegetais hidrogenadas, margarinas sólidas ou cremosas, cremes vegetais, biscoitos e bolachas, sorvetes cremosos, pães, batatas fritas comerciais preparadas em fast food, pastéis, bolos, tortas, massas ou qualquer outro alimento que contenha gordura vegetal hidrogenada entre seus ingredientes $5,6,7$.

O consumo de calorias provenientes de ácidos graxos trans tem sido associado ao aumen- 
to de LDL-colesterol sérico (low density lipoprotein cholesterol) e redução no HDL-colesterol sérico (high density lipoprotein cholesterol), resultando em aumento da relação LDL/HDL. Há evidências de que dietas ricas em ácidos graxos trans podem elevar também as concentrações plasmáticas de triacilgliceróis e de lipoproteína Lp(a) 5,6.

Desta forma, o consumo elevado de ácidos graxos trans pode ser um importante fator de risco para as doenças do coração ${ }^{5}$. Com base em estudos epidemiológicos recentes, a Organização Mundial da Saúde (OMS) recomenda que o consumo máximo deste tipo de gordura não deve ser superior a $1 \%$ das calorias totais 8 .

O objetivo do presente estudo foi avaliar o consumo de ácidos graxos trans e sua relação com os lipídios séricos na comunidade nipo-brasileira de Bauru, São Paulo, Brasil. Comparações entre populações que se deslocaram para regiões com diferentes hábitos alimentares, mas com morbidade e perfil de mortalidade distintos do país de origem (todavia, compartilham a mesma origem genética) podem prover base racional para investigações sobre dieta, estado nutricional e enfermidades crônicas não transmissíveis.

\section{Métodos}

\section{População de estudo}

Na Cidade de Bauru, Estado de São Paulo, desde 1993 pesquisadores do Departamento de Medicina Preventiva, Universidade Federal de São Paulo e de outras instituições vêm desenvolvendo o projeto de pesquisa Diabetes Mellitus na Comunidade Nipo-brasileira de Bauru. Uma das hipóteses de investigação do grupo de estudo consistiu em testar a associação entre as alterações de estilo de vida (hábitos alimentares e atividade física de lazer, especialmente) e a alta prevalência de diabetes e doenças associadas 9,10 .

Em 1993 foi realizado o primeiro estudo transversal. Nesta fase inicial do estudo, $647 \mathrm{ja}$ poneses de primeira $(37,3 \%)$ e segunda geração $(62,7 \%)$, de ambos os gêneros, foram submetidos ao Inquérito de Saúde e Nutrição, conforme descrito em publicação anterior 11. Destes, $69(10,7 \%)$ foram a óbito, $57(8,8 \%)$ mudaram de endereço e 127 (19,6\%) recusaram participar da segunda fase do projeto. Em 2000, um segundo inquérito transversal foi realizado incluindo 394 participantes da primeira fase do estudo (60,9\% do total avaliado em 1993).

Para a presente análise, foram utilizados os dados de consumo alimentar individual e da- dos bioquímicos de 315 nipo-brasileiros de primeira (issei) e segunda (nisei) geração, não miscigenados, de ambos os gêneros, idade entre 40 a 79 anos em 1993, residentes em Bauru, Estado de São Paulo, e que participaram dos dois inquéritos de saúde e nutrição realizados em 1993 e 2000. Dos 394 indivíduos avaliados em 1993 e 2000, foram excluídos 79 participantes (20,1\% do total avaliado em 1993) que não apresentaram informações completas sobre o consumo alimentar ou níveis de lipídios séricos nos dois inquéritos transversais. Quando comparados os indivíduos que participaram dos dois inquéritos $(n=315)$ com os que não foram avaliados em $2000(n=332)$, não houve diferenças estatisticamente significantes segundo gênero, idade e geração.

O estudo foi aprovado pelo Comitê de Ética da Escola Paulista de Medicina, Universidade Federal de São Paulo, com esclarecimento e assinatura do termo de consentimento obtido de todos os participantes.

\section{Avaliação antropométrica e bioquímica}

Medidas de peso (em quilogramas) e altura (em metros) foram obtidas em balança digital (capacidade $150 \mathrm{~kg}$, precisão $100 \mathrm{~g}$ ) e em estadiômetro portátil, respectivamente. O Índice de Massa Corporal (IMC) foi obtido dividindo-se o peso pela estatura ao quadrado $\left(\mathrm{kg} / \mathrm{m}^{2}\right)$. A circunferência da cintura foi obtida com uso de fita métrica inextensível utilizando-se como ponto de referência o plano horizontal na altura da cicatriz umbilical, com o paciente em pé, abdome relaxado, os braços ao longo do corpo e pés unidos. Para avaliação de sobrepeso e obesidade e classificação da obesidade central foram considerados os pontos de corte propostos pela OMS 8

A pressão arterial foi medida três vezes, com o indivíduo sentado após dez minutos de descanso, com aparelho automático (modelo HEM - 712C; Omron Healthcare Inc., Vernon Hills, Estados Unidos). A média das duas últimas medidas foi usada para expressar os níveis pressóricos.

Amostras de sangue foram obtidas após dez horas de jejum, centrifugadas e analisadas imediatamente após a coleta. Concentração de glicose plasmática e níveis séricos de triglicérides, colesterol total e frações foram analisados por processos enzimáticos devidamente padronizados nos dois momentos do estudo, utilizando-se analisador automático (Cobas-mira plus ${ }^{\circledR}$, Roche) 12 . Os pontos de corte adotados para a classificação de dislipidemia foram os propostos pelo National Cholesterol Education 
Program 13, e para diagnóstico de diabetes foram os propostos pela OMS 14 .

\section{Avaliação do consumo alimentar}

Em 1993, o consumo alimentar foi avaliado por meio de um questionário de freqüência alimentar (QFA) adaptado do QFA de Tsunehara et al. 15 com 177 itens alimentares. Em 2000, a dieta habitual foi avaliada por questionário quantitativo de freqüência alimentar (QQFA), com 122 itens alimentares e com reprodutibilidade e validade previamente testados na população japonesa migrante de São Paulo 16,17. Nos dois inquéritos, a avaliação da dieta considerou o consumo habitual de alimentos e bebidas relativo ao período de um ano anterior ao momento da entrevista alimentar. Para padronização da avaliação do consumo alimentar nos dois inquéritos, os dados do QFA de 1993 foram transcritos para o modelo do QQFA utilizado em 2000. A lista de alimentos utilizadas no QFA de 1993 foi mantida no questionário de 2000. Para classificação das porções alimentares do QFA de 1993 (obtidas como variável contínua em gramas ou mililitros) utilizou-se a definição dos tamanhos das porções, como variável categórica (pequena, média, grande e extragrande), conforme modelo adotado no QQFA de 2000.

A descrição da dieta habitual da população de estudo considerou a participação relativa diária de nutrientes selecionados. Os teores de ácidos graxos trans de alimentos como a batata frita, biscoito e sorvete foram calculados com base em tabelas nacionais 7 . A estimativa desse tipo de gordura dos outros alimentos do QFA utilizou tabelas internacionais 18,19. Para alguns alimentos e preparações nacionais não identificados em tabelas publicadas, o teor de ácidos graxos trans foi estimado com base no teor desse tipo de gordura de outro alimento com composição química aproximada: pizza, tortas, pastéis e salgadinhos fritos foram substituídos por folhados; queijos brancos por cheese spread; queijos amarelos por cheese cheddar; requeijão por cheese food american; pão integral e broa de milho por pão branco; castanhas por manteiga de amendoim; tortas doces por bolos; pudim por leite. Para certas preparações foram consideradas preparações padrão: pizza de queijo com $35,6 \%$ deste ingrediente; yakisoba com $20 \%$ de carne; pratos com frango com $80 \%$ deste ingrediente; hot dog com $50 \%$ de pão branco e $50 \%$ de salsicha; salada de maionese com $20 \%$ de maionese. Na Tabela 1 são apresentados os teores de ácidos graxos trans em alimentos e/ou preparações utilizados no presente estudo.
A análise nutricional das dietas foi realizada com auxílio do programa Dietsys 4.01 (National Cancer Institute, Bethesda, Estados Unidos), com dados de composição química de alimentos compilados das tabelas oficiais do Brasil, Japão e Estados Unidos, segundo metodologia descrita em trabalho anterior 17.

\section{Análise estatística}

Para descrição das características gerais da população de estudo foram calculadas médias e desvios-padrão segundo gênero. A análise das diferenças no consumo de nutrientes e dos níveis de lipídios séricos no período empregou teste t de Student para amostras dependentes.

Modelos de regressão linear múltiplos foram utilizados para avaliar a associação entre a diferença no consumo de ácidos graxos trans e a diferença nos valores de lipídios séricos entre 2000 e 1993. Todas as variáveis das diferenças de consumo alimentar foram ajustadas pelas calorias totais pelo método residual, após transformação logarítmica das variáveis que não apresentavam distribuição normal. As diferenças dos lipídios séricos foram consideradas variáveis dependentes. As variáveis independentes foram selecionadas mediante pressupostos teóricos e resultados de estudos anteriores, utilizando-se: (a) variáveis contínuas = diferenças no consumo de ácidos graxos trans, de fibras, de gorduras totais, de gorduras saturadas e de circunferência de cintura, idade em 1993, circunferência de cintura em 1993; (b) variáveis categóricas = gênero, consumo habitual de álcool em 1993 (sim/não), tabagismo em 1993 (sim/não) e presença de morbidade em 1993 (não/sim - indivíduos com diabetes, hipertensão ou em uso de medicamentos que interferem no metabolismo da glicose e lipídios). As análises estatísticas foram conduzidas no programa SPSS versão 10.0 (SPSS Inc., Chicago, Estados Unidos), adotando-se como significantes valores onde $\mathrm{p}<0,05$.

\section{Resultados}

A Tabela 2 apresenta as características gerais da população de estudo em 1993, segundo gênero. A proporção de indivíduos issei/nisei, prevalência de tabagismo e valores médios etários e IMC foram semelhantes entre os gêneros. No entanto, maiores freqüências de consumo habitual de álcool foram observadas entre os homens $\left(\chi^{2}=80,01 ; p=0,00\right)$. Homens e mulheres apresentaram valores médios de IMC dentro do intervalo de eutrofia; porém, esses valores estavam muito próximos do ponto de corte pa- 
Concentração de ácidos graxos trans em alimentos ou preparações do questionário de freqüência alimentar (QQFA) e seus respectivos alimentos correspondentes identificada em tabelas de composição de alimentos (alimento referência).

\begin{tabular}{|c|c|c|}
\hline $\begin{array}{l}\text { Alimento/preparação } \\
\text { do QQFA }\end{array}$ & Alimento referência & $\begin{array}{l}\text { Teor de ácidos } \\
\text { graxos trans } \\
\text { (g/100g de alimento) }\end{array}$ \\
\hline Óleo para salada & Oils: salad and cooking (home use)* & 3,59 \\
\hline Maionese & Mayonnaise* & 1,13 \\
\hline Biscoitos & Biscoito cream cracker, biscoito recheado de chocolate e morango** & 3,76 \\
\hline Bolos & Pastries: doughnuts or piecrusts* & 8,18 \\
\hline Margarina light & Margarine: diet* & 15,30 \\
\hline Margarina & Margarine: stick, margarine: tub or soft* & 22,63 \\
\hline Manteiga & Butter* & 3,37 \\
\hline Requeijão & $\begin{array}{l}\text { Dairy products, cheese food, American, pasteurized } \\
\text { process, brand } a ; c^{\star \star \star}\end{array}$ & 0,71 \\
\hline Chocolate & Candy* & 11,72 \\
\hline Tortas salgadas/Doces & Pastries: doughnuts or piecrusts* & 8,18 \\
\hline Doces/Sobremesas & Candy* & 11,72 \\
\hline Pipoca & $\begin{array}{l}\text { Snacks, popcorn, microwave-popped, brand } a \text {; } b \text {; snacks, } \\
\text { popcorn, microwave-popped. Low fat, brand } a \text {; snacks, popcorn, } \\
\text { oil-popped, brand } a ; b^{\star \star \star}\end{array}$ & 7,32 \\
\hline Salgadinhos em geral & Snacks chips: corn and mixed grains; snack chips: potato* & 11,91 \\
\hline
\end{tabular}

* Enig et al. 18

** Chiara et al. 7 .

$\star \star *$ Exler et al. 19.

Tabela 2

Caracterização da população de estudo em 1993, segundo gênero.

\begin{tabular}{lcc}
\hline & Mulheres $(\mathbf{n}=165)$ & Homens $(\mathbf{n}=\mathbf{1 5 0})$ \\
\hline Proporção issei/nisei & 0,48 & 0,50 \\
Idade média (em anos) & $57,4(\mathrm{DP}=9,9)$ & $56,9(\mathrm{DP}=9,9)$ \\
Índice de massa corporal médio $\left(\mathrm{em} \mathrm{kg} / \mathrm{m}^{2}\right)$ & $24,6(\mathrm{DP}=3,2)$ & $24,7(\mathrm{DP}=3,9)$ \\
Circunferência de cintura média $(\mathrm{em} \mathrm{cm})$ & $83,5(\mathrm{DP}=11,4)$ & $88,4(\mathrm{DP}=9,2)$ \\
Tabagismo & & $88,0 \%$ \\
$\quad$ Não & $81,2 \%$ & $12,0 \%$ \\
$\quad$ Sim & $18,8 \%$ & $59,3 \%$ \\
Consumo habitual de álcool & $12,1 \%$ & $19,3 \%$ \\
Prevalência diabetes & $21,8 \%$ & $90,0 \%$ \\
Prevalência dislipidemia & $88,5 \%$ & \\
\hline
\end{tabular}

ra sobrepeso. Não houve diferença estatisticamente significante para a prevalência de sobrepeso/obesidade entre mulheres e homens, respectivamente (dados não apresentados). Os valores médios da circunferência de cintura apresentaram-se na faixa de risco reduzido para complicações metabólicas entre os homens e de risco elevado/muito elevado entre as mulheres (exceção para as mulheres de primeira geração em 2000). As altas freqüências de diabetes e de dislipidemia foram semelhantes entre homens e mulheres.

A Tabela 3 apresenta valores médios (desvios padrão), valores mínimos e máximos e percentagens das diferenças médias do consumo alimentar e de lipídios séricos no período de estudo, segundo gênero. Com exceção do consumo de proteínas (em gramas) - que redu- 
Valores médios (desvio padrão), mínimos e máximos e percentagens das diferenças médias do consumo alimentar e de lipídios séricos em 1993 e 2000, segundo gênero.

\begin{tabular}{|c|c|c|c|c|c|c|c|c|c|c|}
\hline & \multicolumn{5}{|c|}{ Mulheres $(n=165)$} & \multicolumn{5}{|c|}{ Homens $(n=150)$} \\
\hline & \multirow[t]{2}{*}{1993} & \multirow[t]{2}{*}{2000} & \multicolumn{3}{|c|}{$\begin{array}{c}\text { Diferença média } \\
\text { (2000-1993) }\end{array}$} & \multirow[t]{2}{*}{1993} & \multirow[t]{2}{*}{2000} & \multicolumn{3}{|c|}{$\begin{array}{c}\text { Diferença média } \\
\text { (2000-1993) }\end{array}$} \\
\hline & & & Mínimo & Máximo & $\%$ & & & Mínimo & Máximo & $\%$ \\
\hline $\begin{array}{l}\text { Calorias totais } \\
\text { (em Kcal) }\end{array}$ & $\begin{array}{c}1.763,9 \\
(D P=609,3)\end{array}$ & $\begin{array}{c}1.841,1 \\
(D P=573,1)\end{array}$ & $-1.973,8$ & $3.081,0$ & 4,4 & $\begin{array}{c}2.170,2 \\
(\mathrm{DP}=660,2)\end{array}$ & $\begin{array}{l}2.207,6 \\
(663,7)\end{array}$ & $-2.322,9$ & $2.155,1$ & 1,7 \\
\hline \multicolumn{11}{|l|}{ Carboidratos } \\
\hline gramas & $\begin{array}{c}237,2 \\
(D P=88,1)\end{array}$ & $\begin{array}{c}243,4 \\
(\mathrm{DP}=83,9)\end{array}$ & $-276,4$ & 373,1 & 2,6 & $\begin{array}{c}281,3 \\
(\mathrm{DP}=87,8)\end{array}$ & $\begin{array}{c}285,9 \\
(D P=89,0)\end{array}$ & $-203,2$ & 305,0 & 1,6 \\
\hline$\%$ VCT & $\begin{array}{c}56,9 \\
(\mathrm{DP}=8,8)\end{array}$ & $\begin{array}{c}54,8 \\
(D P=7,6)\end{array}$ & $-25,6$ & 27,9 & $-3,7^{\star}$ & $\begin{array}{c}55,2 \\
(D P=10,2)\end{array}$ & $\begin{array}{c}54,0 \\
(\mathrm{DP}=8,0)\end{array}$ & $-29,1$ & 33,1 & $-2,2$ \\
\hline \multicolumn{11}{|l|}{ Proteínas } \\
\hline gramas & $\begin{array}{c}67,2 \\
(D P=28,8)\end{array}$ & $\begin{array}{c}64,3 \\
(\mathrm{DP}=22,6)\end{array}$ & $-137,0$ & 138,0 & $-4,3$ & $\begin{array}{c}85,1 \\
(\mathrm{DP}=39,8)\end{array}$ & $\begin{array}{c}73,6 \\
(D P=23,4)\end{array}$ & $-273,7$ & 63,4 & $-13,5^{\star \star}$ \\
\hline$\%$ VCT & $\begin{array}{c}16,1 \\
(\mathrm{DP}=4,0)\end{array}$ & $\begin{array}{c}14,4 \\
(D P=2,4)\end{array}$ & $-22,9$ & 8,63 & $-10,6^{*}$ & $\begin{array}{c}16,3 \\
(\mathrm{DP}=4,2)\end{array}$ & $\begin{array}{c}13,9 \\
(D P=2,8)\end{array}$ & $-27,8$ & 7,4 & $-14,7^{\star}$ \\
\hline \multicolumn{11}{|l|}{ Lipídios } \\
\hline gramas & $\begin{array}{c}52,2 \\
(D P=23,1)\end{array}$ & $\begin{array}{c}63,1 \\
(D P=23,0)\end{array}$ & $-80,0$ & 84,7 & $20,9 *$ & $\begin{array}{c}60,5 \\
(D P=25,5)\end{array}$ & $\begin{array}{c}73,5 \\
(D P=30,4)\end{array}$ & $-78,4$ & 183,5 & $21,5^{\star}$ \\
\hline$\%$ VCT & $\begin{array}{c}28,0 \\
(\mathrm{DP}=6,5)\end{array}$ & $\begin{array}{c}31,9 \\
(D P=6,5)\end{array}$ & $-18,0$ & 25,6 & $14,3^{*}$ & $\begin{array}{c}26,2 \\
(\mathrm{DP}=6,3)\end{array}$ & $\begin{array}{c}30,7 \\
(D P=6,5)\end{array}$ & $-15,4$ & 28,0 & $18,6^{*}$ \\
\hline \multicolumn{11}{|l|}{ Gordura saturada } \\
\hline gramas & $\begin{array}{c}15,6 \\
(D P=7,1)\end{array}$ & $\begin{array}{c}15,0 \\
(D P=6,2)\end{array}$ & $-3,17$ & 23,2 & $-3,2$ & $\begin{array}{c}18,2 \\
(\mathrm{DP}=8,1)\end{array}$ & $\begin{array}{c}17,8 \\
(D P=7,6)\end{array}$ & $-32,9$ & 23,0 & $-2,2$ \\
\hline$\%$ VCT & $\begin{array}{c}8,4 \\
(D P=2,3)\end{array}$ & $\begin{array}{c}7,6 \\
(D P=2,0)\end{array}$ & $-7,6$ & 6,5 & $-9,5$ & $\begin{array}{c}7,9 \\
(D P=2,4)\end{array}$ & $\begin{array}{c}7,4 \\
(D P=2,0)\end{array}$ & $-7,2$ & 6,8 & $-6,3$ \\
\hline \multicolumn{11}{|l|}{ Ácidos graxos trans } \\
\hline gramas & $\begin{array}{c}10,2 \\
(\mathrm{DP}=6,5)\end{array}$ & $\begin{array}{c}7,0 \\
(\mathrm{DP}=4,8)\end{array}$ & $-23,9$ & 30,8 & $-30,4^{\star}$ & $\begin{array}{c}11,5 \\
(\mathrm{DP}=7,4)\end{array}$ & $\begin{array}{c}8,3 \\
(D P=5,1)\end{array}$ & $-25,7$ & 19,7 & $-27,8^{\star}$ \\
\hline$\%$ VCT & $\begin{array}{c}5,1 \\
(\mathrm{DP}=2,6)\end{array}$ & $\begin{array}{c}3,4 \\
(\mathrm{DP}=1,7)\end{array}$ & $-14,8$ & 4,0 & $-35,3^{*}$ & $\begin{array}{c}4,7 \\
(D P=2,4)\end{array}$ & $\begin{array}{c}3,3 \\
(D P=1,9)\end{array}$ & $-8,7$ & 11,3 & $-29,8^{\star}$ \\
\hline Colesterol (mg) & $\begin{array}{c}219,7 \\
(D P=120,8)\end{array}$ & $\begin{array}{c}162,0 \\
(D P=78,8)\end{array}$ & $-694,4$ & 470,0 & $-26,2^{\star}$ & $\begin{array}{c}278,0 \\
(\mathrm{DP}=160,2)\end{array}$ & $\begin{array}{c}211,1 \\
(D P=115,8)\end{array}$ & $-749,2$ & 286,6 & $-24,1^{\star}$ \\
\hline Fibras (g) & $\begin{array}{c}17,0 \\
(\mathrm{DP}=8,8)\end{array}$ & $\begin{array}{c}17,3 \\
(\mathrm{DP}=8,1)\end{array}$ & $-50,6$ & 19,1 & 1,8 & $\begin{array}{c}18,2 \\
(\mathrm{DP}=7,9)\end{array}$ & $\begin{array}{c}19,1 \\
(\mathrm{DP}=8,4)\end{array}$ & $-20,7$ & 24,1 & 4,9 \\
\hline \multicolumn{11}{|l|}{ Níveis séricos (mg/dL) } \\
\hline Colesterol & $\begin{array}{c}216,2 \\
(D P=43,9)\end{array}$ & $\begin{array}{c}218,5 \\
(\mathrm{DP}=39,2)\end{array}$ & $-140,5$ & 115,5 & 1,0 & $\begin{array}{c}211,8 \\
(\mathrm{DP}=43,5)\end{array}$ & $\begin{array}{c}207,8 \\
(\mathrm{DP}=40,9)\end{array}$ & $-161,5$ & 155,0 & $-1,7$ \\
\hline LDL colesterol & $\begin{array}{c}150,7 \\
(\mathrm{DP}=78,8)\end{array}$ & $\begin{array}{c}132,9 \\
(\mathrm{DP}=36,5)\end{array}$ & $-883,0$ & 94,0 & $-10,4^{\star}$ & $\begin{array}{c}139,6 \\
(\mathrm{DP}=35,7)\end{array}$ & $\begin{array}{c}121,5 \\
(\mathrm{DP}=36,0)\end{array}$ & $-104,5$ & 62,5 & $-11,5^{\star}$ \\
\hline HDL colesterol & $\begin{array}{c}44,3 \\
(\mathrm{DP}=21,7)\end{array}$ & $\begin{array}{c}51,5 \\
(\mathrm{DP}=10,2)\end{array}$ & $-220,0$ & 90,5 & 16,0 * & $\begin{array}{c}38,7 \\
(D P=10,0)\end{array}$ & $\begin{array}{c}48,9 \\
(D P=13,3)\end{array}$ & $-26,0$ & 78,5 & $24,8^{*}$ \\
\hline Triglicerídios séricos & $\begin{array}{c}157,6 \\
(D P=94,6)\end{array}$ & $\begin{array}{c}224,0 \\
(D P=165,6)\end{array}$ & $-461,0$ & $1.120,5$ & $43,9 *$ & $\begin{array}{c}213,5 \\
(D P=211,7)\end{array}$ & $\begin{array}{c}308,2 \\
(\mathrm{DP}=295,9)\end{array}$ & $-1.572,0$ & $1.543,0$ & $46,3^{*}$ \\
\hline
\end{tabular}

$\mathrm{VCT}=$ Valor calórico total.

*Teste $t$ de Student para amostras dependentes, $p<0,01$

**Teste $t$ de Student para amostras dependentes, $p<0,05$. 
ziu entre os homens e se manteve entre as mulheres -, e do percentual calórico proveniente dos carboidratos - que reduziu entre as mulheres e se manteve entre os homens -, a mudança no consumo dos nutrientes foi semelhante entre os gêneros. Não se observou alteração no consumo das calorias totais; contudo, houve redução estatisticamente significante na contribuição percentual calórica das proteínas e aumento deste percentual proveniente das gorduras totais. Entre os componentes dos lipídios ingeridos, o aumento no consumo das gorduras totais ocorreu essencialmente devido ao maior consumo de ácidos graxos polinsaturados. Não ocorreu alteração no consumo de gordura saturada, com redução no consumo de ácidos graxos trans e de colesterol. A ingestão de fibras não apresentou alteração significante. As mudanças no perfil de lipídios séricos foram semelhantes para ambos os gêneros: constatou-se redução dos níveis séricos de LDL colesterol com aumento estatisticamente significante do HDL colesterol e triglicerídeos.

Na Tabela 4 podem ser observados os valores para os coeficientes de regressão $\beta_{1}$ (inter-

Tabela 4

Coeficientes de regressão $\beta_{1}$ (IC95\%) em modelo linear múltiplo entre a diferença de consumo dos ácidos graxos trans (variável independente) e as diferenças dos níveis de lipídios séricos (variáveis dependentes).

\begin{tabular}{|c|c|c|c|}
\hline \multirow[t]{2}{*}{$\begin{array}{l}\text { Variáveis dependentes } \\
\text { níveis séricos }\end{array}$} & \multicolumn{3}{|c|}{$\begin{array}{l}\text { Diferença do consumo } \\
\text { de ácidos graxos trans* }\end{array}$} \\
\hline & $\beta_{1}$ & IC95\% & $p$ \\
\hline \multicolumn{4}{|l|}{ Colesterol sérico total } \\
\hline Ajuste gênero e idade & $-0,115$ & $-0,883-0,653$ & 0,769 \\
\hline Ajuste múltiplo** & $-0,149$ & $-0,935-0,638$ & 0,710 \\
\hline \multicolumn{4}{|l|}{ LDL-colesterol sérico* } \\
\hline Ajuste gênero e idade & $-0,00071$ & $-0,004-0,002$ & 0,625 \\
\hline Ajuste múltiplo** & $-0,00129$ & $-0,004-0,002$ & 0,395 \\
\hline \multicolumn{4}{|l|}{ HDL-colesterol sérico* } \\
\hline Ajuste gênero e idade & $-0,00049$ & $-0,003-0,002$ & 0,676 \\
\hline Ajuste múltiplo** & $-0,00024$ & $-0,003-0,002$ & 0,843 \\
\hline \multicolumn{4}{|l|}{ Triglicérides sérico* } \\
\hline Ajuste gênero e idade & $-0,00101$ & $-0,005-0,003$ & 0,656 \\
\hline Ajuste múltiplo** & $-0,00164$ & $-0,006-0,003$ & 0,489 \\
\hline \multicolumn{4}{|c|}{$\begin{array}{l}\text { * Dados que sofreram transformação logarítmica. } \\
\text { ** Variáveis contínuas: diferença no consumo de ácidos graxos trans, de fibras, } \\
\text { de gorduras totais, de gorduras saturadas, de circunferência de cintura, e idade } \\
\text { e circunferência de cintura em 1993. Variáveis categóricas: gênero (feminino } \\
\text { e masculino), consumo habitual de álcool em } 1993 \text { (sim e não), tabagismo em } 1993 \\
\text { (sim e não) e presença de morbidade em } 1993 \text { (sim e não; indivíduos com diabetes, } \\
\text { hipertensão ou em uso de medicamentos que interferem no metabolismo } \\
\text { da glicose e lipídios). }\end{array}$} \\
\hline
\end{tabular}

valo com 95\% de confiança - IC95\%) entre a diferença de consumo dos ácidos graxos trans e as diferenças dos níveis de lipídios séricos. Não houve associação estatisticamente significante entre consumo de ácidos graxos trans e lipídios séricos. No entanto, para as demais variáveis independentes consideradas nos modelos, observou-se associação inversa somente entre a diferença no consumo de fibras da dieta com o colesterol sérico total na população estudada $\left(\beta_{1}=-1,377\right.$; IC95\%: -2,172- -0,582).

\section{Discussão}

No presente estudo, o consumo das calorias totais da dieta habitual no período estudado situou-se na faixa da recomendação específica para japoneses em ambos os gêneros 20 . Apesar do consumo de gorduras saturadas e de colesterol se encontrarem de acordo com as recomendações dietéticas, o consumo de ácidos graxos trans estimado foi muito acima do recomendado: a OMS preconiza que o consumo desse tipo de gordura deve ser menor que $1 \%$ das calorias totais da dieta 8 .

Em 1994, Willett \& Ascherio 21 destacaram os efeitos adversos das gorduras trans e estimaram que mais de 30 mil mortes por ano nos Estados Unidos poderiam ser atribuídas ao consumo de gordura parcialmente hidrogenada. A hidrogenação parcial de óleos vegetais é utilizada para produzir gorduras sólidas em temperatura ambiente. A adição de moléculas de hidrogênio nas duplas ligações reduz a concentração de ácidos graxos polinsaturados essenciais (linolênico e linoleico). Estima-se que o efeito dos ácidos graxos trans na razão LDL/ HDL colesterol séricos seja cerca do dobro do esperado para ácidos graxos saturados 6 .

No entanto, até o presente momento, evidências epidemiológicas sobre a associação inversa entre gordura trans da dieta e HDL colesterol ou associação positiva com LDL colesterol são ainda controversas.

Um estudo prospectivo com 14 anos de seguimento de 43.732 homens americanos não encontrou associação entre consumo de gordura total, tipo de gordura ou colesterol e risco de acidente vascular isquêmico e hemorrágico 22. Entretanto, em outro estudo prospectivo, Nurse's Health Study, encontrou-se que mulheres com consumo de grandes quantidades de ácidos graxos trans (3\% das calorias totais diárias) apresentavam $50 \%$ mais chances de desenvolver doenças do coração que aquelas que consumiam pequenas quantidades deste tipo de gordura (aproximadamente $1 \%$ das calorias 
totais diárias). Mulheres com um consumo baixo de ácidos graxos trans e de ácidos graxos polinsaturados apresentaram $70 \%$ menos chance de desenvolver doenças do coração quando comparadas a mulheres que consumiam muito ácidos graxos trans e pouca gordura polinsaturada 4 . Outros estudos de coorte também encontraram associação positiva entre alto consumo de ácidos graxos trans e risco para doenças coronarianas em ambos os gêneros 1,2,3,4.

No presente estudo, observou-se redução estatisticamente significante no consumo total de gordura trans, acompanhado de redução dos níveis séricos de LDL colesterol e aumento do HDL colesterol no intervalo de sete anos de seguimento. Porém, não houve associação entre a diferença do consumo habitual de ácidos graxos trans e alteração dos níveis de lipídios séricos. Resultado semelhante foi descrito por van de Vijver et al. 23 em estudo transversal com 327 homens e 299 mulheres europeus: não foi observada associação entre consumo de ácidos graxos trans e níveis séricos de LDL colesterol e HDL colesterol, mas foi encontrada uma fraca associação inversa entre consumo de ácidos graxos trans e colesterol sérico total.

\section{Resumo}

A influência da alteração do consumo de ácidos graxos trans nos níveis de lipídios séricos foi avaliada em 328 nipo-brasileiros de primeira (issei) e segunda geração (nisei), entre 40 e 79 anos, em 1993, participantes de dois inquéritos de saúde e nutrição realizados em 1993 e 2000. A relação entre a diferença do consumo de ácidos graxos trans e a diferença entre os níveis de lipídios séricos entre os dois inquéritos foi analisada em modelos de regressão linear múltiplos. Em ambos os gêneros, observou-se redução estatisticamente significante no consumo total de gordura trans, acompanhado de redução dos níveis séricos de LDL colesterol e aumento do HDL colesterol em sete anos de seguimento. Os valores médios de ingestão de gordura trans (percentual das calorias totais) em 1993 e 2000 foram: $5,1 \%$ e 3,4\% para mulheres e 4,7\% e 3,3\% para homens, respectivamente. Apesar da ausência de associação entre as alterações do consumo de ácidos graxos trans e alterações no perfil de lipídios séricos, o nível de ingestão de gordura trans foi acima do recomendado pela OMS (até 1\% das calorias totais).

Ácidos Graxos Trans; Lipídios; Consumo de Alimentos
Uma das limitações do presente estudo pode estar relacionada à estimativa do consumo de ácidos graxos trans, calculada com auxílio de tabela internacional de acordo com procedimentos adotados em estudos anteriores internacionais. Erros aleatórios na medida do consumo deste tipo de gordura pode ter resultado em subestimativa da associação com as alterações observadas nos lipídios séricos 6 . Investigação recente sobre teores de ácidos graxos trans em alimentos brasileiros selecionados sugere concentrações maiores desse tipo de gordura em relação ao esperado segundo informações de tabelas ou de rótulos dos alimentos, recomendando-se regulamentação mais cautelosa para produtos alimentícios com gordura hidrogenada 7.

Com base nos resultados apresentados neste estudo, apesar da ausência de associação entre alterações do consumo de ácidos graxos trans e alterações no perfil de lipídios séricos em estudo prospectivo, o nível de ingestão desse tipo de gordura em nipo-brasileiros foi acima dos valores preconizados pela OMS para prevenção de doenças cardiovasculares, sugerindo a necessidade de monitoramento dos teores de gordura trans na dieta habitual da população brasileira.

\section{Colaboradores}

C. N. Bertolino realizou revisão da literatura, estimativa e cálculo do consumo de ácidos graxos trans, processamento e análise dos dados e elaboração do manuscrito. T. G. Castro e D. S. Sartorelli colaboraram na análise dos dados e leitura crítica do manuscrito. S. R. G. Ferreira coordenou e desenvolveu o trabalho de campo juntamente com o Grupo de Estudos de Diabetes em Nipo-brasileiros. M. A. Cardoso orientou todas as etapas do estudo e elaboração da versão final do artigo.

\section{Outros membros do Grupo de Estudos de Diabetes em Nipo-brasileiros}

N. Barros Jr., M. A. Cardoso, R. Chaim, V. D'Almeida, S. R. G. Ferreira, L. J. Franco, R. D. Freire, S. G. A. Gimeno, H. Harima, A. Hirai, A. T. Hirai, M. Iunes, M. Kikuchi, L. Matsumura, R. S. Moisés, K. Osiro, N. Tomita, K. Wakisaka.

\section{Agradecimentos}

C. N. Bertolino foi bolsista de iniciação científica do Conselho Nacional de Desenvolvimento Científico e Tecnológico (CNPq). 


\section{Referências}

1. Hu FB, Stampfer MJ, Manson JE, Rimm E, Colditz GA, Rosner BA, et al. Dietary fat intake and the risk of coronary heart disease in women. $\mathrm{N}$ Engl J Med 1997; 337:1491-9.

2. Hu FB, Stampfer MJ, Rimm E, Ascherio A, Rosner BA, Spiegelman D, et al. Dietary fat and coronary heart disease: a comparison of approaches for adjusting for total energy intake and modeling repeated dietary measurements. Am J Epidemiol 1999; 149:531-40.

3. Pietinen P, Ascherio A, Korhonen P, Hartman AM, Willett WC, Albanes D, et al. Intake of fatty acids and risk of coronary heart disease in a cohort of Finnish men. The Alpha-Tocopherol, Beta-Carotene Cancer Prevention Study. Am J Epidemiol 1997; 145:876-87.

4. Willett WC, Stampfer MJ, Manson JE, Colditz GA, Speizer FE, Rosner BA, et al. Intake of trans fatty acids and risk of coronary heart disease among women. Lancet 1993; 341:581-5.

5. Willett WC. Surprising news about fat. In: Willett WC, editor. Eat, drink and be healthy: the Harvard Medical School guide to healthy eating. New York: Simon \& Schuster Adult Publishing Group; 2001. p. 56-84.

6. Ascherio A, Katan MB, Zock PL, Stampfer MJ, Willett WC. Trans fatty acids and coronary heart disease. N Engl J Med 1999; 340:1994-8.

7. Chiara VL, Sichieri R, Carvalho TSF. Teores de ácidos graxos trans de alguns alimentos consumidos no Rio de Janeiro. Rev Nutr 2003; 16:227-33.

8. World Health Organization/Food and Agricultural Organization. Diet, nutrition and the prevention of chronic disease. Geneva: World Health Organization; 2003. (WHO Technical Report Series 916).

9. Ferreira SRG, Iunes M, Franco LJ, Iochida LC, Hirai A, Vivolo MA. Disturbances of glucose and lipid metabolism in first and second generation Japanese-Brazilians. Diabetes Res Clin Pract 1996; 34 Suppl:S59-63.

10. Franco LJ. Diabetes in Japanese-Brazilians - influence of the acculturation process. Diabetes Res Clin Pract 1996; 34 Suppl:S51-7.

11. Gimeno SGA, Ferreira SRG, Cardoso MA, Franco LJ, Iunes M. Weight gain in adulthood and risk of developing glucose tolerance disturbance: a study of a Japanese-Brazilian population. JapaneseBrazilian Diabetes Study Group. J Epidemiol 2000; 10:103-10.

12. Friedwald W, Levy RJ, Friedrickson DS. Estimation of the concentration of low-density lipoprotein cholesterol in plasma, without use of the preparative ultracentrifuge. Clin Chem 1972; 18: 499-502.
13. Expert Panel on Detection, Evaluation, and Treatment of High Blood Cholesterol in Adults. Executive Summary of the Third Report of The National Cholesterol Education Program (NCEP) Expert Panel on Detection, Evaluation, and Treatment of High Blood Cholesterol in Adults (Adult Treatment Panel III). JAMA 2001; 285:2486-97.

14. Alberti KG, Zimmet PZ. Definition, diagnosis and classification of diabetes mellitus and its complications. Part 1: diagnosis and classification of diabetes mellitus provisional report of a WHO consultation. Diabet Med 1998; 15:539-53.

15. Tsunehara CH, Leonetti DL, Fujimoto WY. Diet of second-generation Japanese-American men with and without non-insulin-dependent diabetes. Am J Clin Nutr 1990; 52:731-8.

16. Cardoso MA, Kida AA, Tomita LY, Stocco PR. Reproducibility and validity of a food frequency questionnaire among women of Japanese ancestry living in Brazil. Nutr Res 2001; 21:725-33.

17. Cardoso MA, Stocco PR. Desenvolvimento de um questionário quantitativo de freqüência alimentar em imigrantes japoneses e seus descendentes residentes em São Paulo, Brasil. Cad Saúde Pública 2000; 16:107-14.

18. Enig MG, Pallansch LA, Sampugna J, Keeney M. Fatty acid composition of the fat in selected food items with emphasis on trans components. J Am Oil Chem Soc 1983; 60:1788-94.

19. Exler J, Lemar L, Smith J. Fat and fatty acid content of selected foods containing trans-fatty acids. http://www.nal.usda.gov/fnic/etext/000020.html (acessado em 20/Ago/2004).

20. Ministry of Health and Welfare. Recommended dietary allowances for the Japanese $-5^{\text {th }}$ revision. Tokyo: Daiichi Shuppan; 1996.

21. Willett WC, Ascherio A. Trans fatty acids: are the effects only marginal? Am J Public Health 1994; 84:722-4.

22. He K, Merchant A, Rimm EB, Rosner BA, Stampfer MJ, Willett WC, et al. Dietary fat intake and risk of stroke in male US healthcare professionals: 14 year prospective cohort study. BMJ 2003; 327:777-82.

23. van de Vivjer LPL, Kardinaal AFM, Couet C, Aro A, Kafatos A, Steingrimsdottir L, et al. Association between trans fatty acid intake and cardiovascular risk factors in Europe: the TRANSFAIR study. Eur J Clin Nutr 2000; 54:126-35.

Recebido em 22/Dez/2004

Versão final reapresentada em 27/Abr/2005 Aprovado em 02/Ago/2005 